\title{
Síndrome de Treacher Collins e alterações fonoaudiológicas: uma revisão narrativa
}

\author{
Treacher Collins syndrome and speech-language disorders: a narrative review \\ Síndrome de Treacher Collins y trastornos del habla y el lenguaje: una revisión narrativa
}

Recebido: 09/03/2021 | Revisado: 16/03/2021 | Aceito: 17/03/2021 | Publicado: 22/03/2021

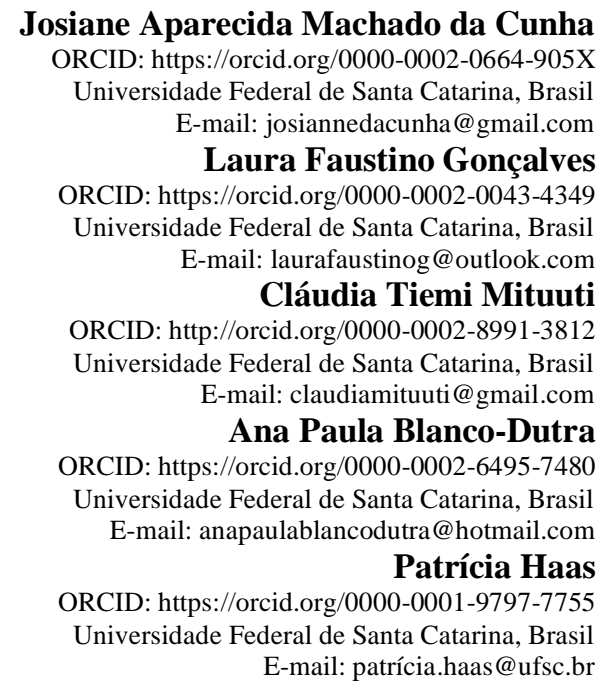

\begin{abstract}
Resumo
Objetivo: Apresentar evidências científicas com base em revisão narrativa, seguindo o Protocolo PRISMA sobre as alterações auditivas, disfágicas e de linguagem em pacientes com a Síndrome de Treacher Collins. Metodologia: A busca de artigos foi realizada nas bases de dados Lilacs, Pubmed, Science Direct, Scielo e Scopus, não houve restrição de localização e idioma, o período avaliado consistiu entre 2015 a 2020. No processo de seleção dos artigos foi utilizada uma combinação baseada no Medical Subject Heading Terms (MeSH). Foram incluídos na pesquisa quatro estudos que obtiveram pontuação $\geq$ a 6 pontos segundo o protocolo para pontuação qualitativa proposto por Pithon et al. (2015). Resultados: Os pacientes acometidos pela síndrome de Treacher Collins podem apresentar complicações nas vias aéreas superiores, micrognatia, alterações anatômicas na orelha externa, dentição e articulação temporomandibular. Conclusão: As características sindrômicas da disostose mandibulofacial afetam diretamente a audição, fala, voz, deglutição e respiração dos pacientes, sendo essas particularidades que tornam a atuação fonoaudiológica na equipe multidisciplinar de extrema importância para o prognóstico e manutenção da qualidade de vida dos pacientes acometidos pela síndrome de Treacher Collins.
\end{abstract}

Palavras-chave: Fonoaudiologia; Disostose Mandibulofacial; Estudos da Fala, Linguagem e Audição.

\begin{abstract}
Objective: To present scientific evidence based on narrative review, following the PRISMA Protocol on auditory, dysphagic and language disorders in patients with Treacher Collins Syndrome. Methodology: The search for articles was carried out in the Lilacs, Pubmed, Science Direct, Scielo and Scopus databases, there was no restriction on location and language, the evaluated period consisted of 2015 to 2020. In the article selection process a combination based on Medical Subject Heading Terms (MeSH) was used. Four studies that scored $\geq 6$ points according to the qualitative score protocol proposed by Pithon et al. (2015). Results: Patients affected by Treacher Collins syndrome may present complications in the upper airways, micrognathia, anatomical changes in the outer ear, dentition and temporomandibular joint. Conclusion: The syndromic characteristics of mandibulofacial dysostosis directly affect the hearing, speech, voice, swallowing and breathing of patients, and these are the particularities that make speech therapy in the multidisciplinary team extremely important for the prognosis and maintenance of the quality of life of patients affected by the syndrome Treacher Collins.
\end{abstract}

Keywords: Speech, language and hearing studies; Mandibulofacial Dysostosis; Speech, language and hearing studies.

\section{Resumen}

Objetivo: Presentar evidencia científica basada en revisión narrativa, siguiendo el Protocolo PRISMA sobre trastornos auditivos, disfágicos y del lenguaje en pacientes con Síndrome de Treacher Collins. Metodología: La búsqueda de artículos se realizó en las bases de datos Lilacs, Pubmed, Science Direct, Scielo y Scopus, no hubo restricción de 
ubicación e idioma, el período evaluado fue de 2015 a 2020. En el proceso de selección de artículos se utilizó una combinación basada en términos del Medical Subject Heading (MeSH). Cuatro estudios que puntuaron $\geq 6$ puntos según el protocolo de puntuación cualitativa propuesto por Pithon et al. (2015). Resultados: Los pacientes afectados por el síndrome de Treacher Collins pueden presentar complicaciones en las vías respiratorias superiores, micrognatia, alteraciones anatómicas del oído externo, dentición y articulación temporomandibular. Conclusión: Las características sindrómicas de la disostosis mandibulofacial inciden directamente en la audición, habla, voz, deglución y respiración de los pacientes, y estas son las particularidades que hacen de la logopedia en el equipo multidisciplinario de suma importancia para el pronóstico y mantenimiento de la calidad de vida de los pacientes afectados el síndrome de Treacher Collins.

Palabras clave: Estudios del habla, lenguaje y audición; Disostosis mandibulofacial; Estudios del habla, lenguaje y audición.

\section{Introdução}

A Síndrome de Treacher Collins, também conhecida como disostose mandibulofacial, é considerada uma condição patológica congênita e pode apresentar impacto na anatomia da face, portanto um agravante nas condições miofasciais do paciente (Massi, et al., 2019). Trata-se de um transtorno morfogenético de herança autossômica dominante e sua incidência é de 1 para cada 50.000 nascidos vivos (Saoud, et al., 2020).

Segundo Duque (2019) os pacientes acometidos por este distúrbio normalmente apresentam condições de hipoplasia facial que os condicionam a apresentar uma face estreita com depressão das bochechas, além de alterações respiratórias como a respiração bucal, apinhamento dental, palato ogival, macrostomia e fenda palatina, além de disfunção causada por displasia ou aplasia das glândulas salivares. Outro aspecto importante de ser ressaltado versa sobre quando a hipoplasia é mais grave em nível proximal, o que indica que o côndilo é a parte mais hipoplásica, causando assim disfunções do funcionamento da articulação temporomandibular o que limita a abertura bucal.

Outro aspecto afetado a ser destacado pela Síndrome de Treacher Collins é o impacto na audição, já que esta condição sindrômica causa deformidades ou ausência nos pavilhões auriculares, diminuindo a acuidade auditiva por conta da hiperplasia do conduto auditivo externo e dos ossículos da orelha média (Silva, 2018). Massi et al. (2016) destacam que a Síndrome de Treacher Collins é uma doença que demonstra a indispensabilidade de atuação fonoaudiológica para avaliação e reabilitação audiológica, de motricidade orofacial, disfagia e linguagem.

A partir do exposto, a presente pesquisa apresenta como objetivo principal e norteador avaliar as alterações auditivas, disfágicas e de linguagem em pacientes com a Síndrome de Treacher Collins, visando responder a seguinte pergunta norteadora de pesquisa: Quais alterações fonoaudiológicas estão associadas a Síndrome de Treacher Collins?

\section{Metodologia}

\section{Protocolo e registro}

A presente revisão narrativa foi conduzida conforme as recomendações PRISMA (Preferred Reporting Items for Systematic reviews and Meta-Analyses) (Moher et al., 2015). As buscas por artigos científicos foram conduzidas por dois pesquisadores independentes nas bases de dados eletrônicas LILACS, Pubmed, Science Direct, Scielo e Scopus, sem restrição de idioma e localização, durante o período de 2015 a 2020. A pesquisa foi estruturada e organizada na forma PICOS, que representa um acrônimo para População alvo, a Intervenção, Comparação, "Outcomes" (desfechos) e Study. População de interesse ou problema de saúde (P) corresponde a pacientes; intervenção (I): ações fonoaudiológicas; comparação (C): associação; outcome (O): Treacher Collins; (S): estudo transversal, estudo observacional, relatos de caso, estudos de casocontrole, ensaios clínicos controlados, estudos de coorte. (Tabela 1). 
Tabela 1. Descrição dos componentes do PICOS.

\begin{tabular}{l|l}
\hline \multicolumn{1}{c|}{ Acrônimo } & \multicolumn{1}{c}{ Definição } \\
\hline $\mathbf{P}$ & Pacientes \\
\hline $\mathbf{I}$ & Ações Fonoaudiológicas \\
\hline $\mathbf{C}$ & Associação \\
\hline $\mathbf{O}$ & Treacher Collins \\
\hline $\mathbf{S}$ & Estudo transversal \\
& Estudo observacional \\
& Relatos de caso \\
& Estudos de caso-controle \\
& Ensaios clínicos controlados \\
& Estudos de coorte \\
\hline
\end{tabular}

Fonte: Desenvolvido pelos autores.

\section{Estratégia de pesquisa}

Os descritores foram selecionados a partir do dicionário Descritores em Ciências da Saúde (DeCS) e Medical Subject Heading Terms (MeSH), haja vista a sua grande utilização pela comunidade científica para a indexação de artigos na base de dados PubMed. Diante da busca dos descritores, foi realizada a adequação para as outras bases utilizadas. Em um primeiro momento foram propostas para as buscas os seguintes descritores e operadores booleanos: (Treacher collins Syndrome) or (mandibulofacial dysostosis) or (speech) or (maxillofacial abnormalities) or (speech therapy). A busca ocorreu em dezembro de 2020 de forma concentrada. Para complementar, foi realizada uma busca por literatura cinza no Google Scholar. Pesquisa de natureza quantitativa.

\section{Critérios de Elegibilidade}

Foram incluídos estudos sem restrição de idioma e localização, entre o período de maior concentração de artigos com o tema norteador deste estudo, 2015 a 2020. A Tabela 2 representa os critérios de inclusão e exclusão de senvolvidos para esta pesquisa. O estudo obteve pontuação 12 no protocolo modificado de Pithon et al. (2015) para avaliação da qualidade dos mesmos. 
Tabela 2. Síntese dos critérios de inclusão/exclusão.

\begin{tabular}{c|c}
\hline \multicolumn{2}{c}{ Critérios de Inclusão } \\
\hline Delineamento & Estudo clínico \\
\hline Localização & Sem Restrição \\
\hline Idioma & Sem restrição \\
\hline \multicolumn{2}{c}{ Critérios de Exclusão } \\
\hline Delineamento & Cartas ao editor \\
& Diretrizes \\
& Revisões de literatura \\
& Revisões sistemáticas \\
Estudos & Meta-análises \\
\hline Forma de publicação & Estudos pouco claros \\
& Mal descritos ou inadequados \\
\hline
\end{tabular}

Fonte: Desenvolvido pelos autores.

\section{Risco de viés}

A qualidade dos métodos utilizados no estudo incluído foi avaliada pelo revisor de forma independente $(\mathrm{PH})$, de acordo com a recomendação PRISMA (Moher et al., 2015). A avaliação priorizou a descrição clara das informações. Neste ponto, a revisão foi realizada às cegas, mascarando os nomes dos autores e revistas, evitando qualquer viés potencial e conflito de interesses.

\section{Critérios de Exclusão}

Foram excluídos estudos publicados no formato de Cartas ao editor, diretrizes, revisões de literatura, revisões sistemáticas, meta análises e resumos. Estudos que não tenham descrito ou que foram pouco claros ou, ainda, indisponíveis na íntegra. Estes critérios estão representados na Tabela 2.

\section{Análise dos dados}

A extração dos dados para o processo de elegibilidade dos estudos foi realizada utilizando-se uma ficha própria para revisão narrativa elaborada por dois pesquisadores em Programa Excel $^{\circledR}$, na qual os dados extraídos foram adicionados por um dos pesquisadores e, então, conferidos por outro pesquisador. Inicialmente foram selecionados de acordo com o título; em seguida, os resumos foram analisados e apenas os que fossem potencialmente elegíveis foram selecionados para a próxima etapa. Com base nos resumos, artigos foram selecionados para leitura integral, foram admitidos os que atendiam a todos os critérios pré-determinados para a presente pesquisa.

\section{Forma de seleção dos estudos}

Inicialmente o revisor de elegibilidade (JC e LFG) foram calibrados para a realização da revisão narrativa por PH. Após a calibração e esclarecimentos de dúvidas, os títulos e resumos foram examinados pelo revisor de elegibilidade (JC e LFG), de forma independente, os quais não estavam cegos para o nome dos autores e das revistas. Aqueles que apresentaram um título dentro do âmbito, mas os resumos não estavam disponíveis, também foram obtidos e analisados na íntegra. Foram excluídos estudos fora do âmbito, relatos de caso, cartas ao editor e/ou editorial, revisões de literatura, índices, resumos e estudos em animais. Sucessivamente, os estudos elegíveis preliminarmente tiveram o texto completo obtido e avaliado. Em 
casos específicos, quando o estudo com potencial de elegibilidade apresentasse dados incompletos, os autores poderiam ser contatados por e-mail para mais informações.

\section{Dados Coletados}

Após a triagem, o texto do artigo selecionado foi revisado e extraído de forma padronizada por dois autores (LFG e JC) sob a supervisão de PH, identificando-se ano de publicação, local da pesquisa, idioma de publicação, tipo de estudo, amostra, método, resultado e conclusão do estudo.

\section{Resultado clínico}

O resultado clínico de interesse consistiu em apresentar evidências científicas com base em revisão narrativa, seguindo o Protocolo PRISMA sobre as alterações auditivas, disfágicas e de linguagem nos pacientes com a Síndrome de Treacher Collins. Aqueles que não utilizaram a abordagem não fizeram parte da amostra da revisão de literatura.

\section{Resultados e Discussão}

Inicialmente foram selecionados 4.082 artigos, dimensionado para 4.081 após exclusão por repetição; em seguida, os títulos e resumos foram analisados e 4.077 trabalhos foram excluídos pois não estavam no escopo da proposta da pesquisa. Sendo então admitidos para a análise final 4 artigos, dos quais todos foram incluídos na presente pesquisa (Figura 1). 
Figura 1. Fluxograma do processo de busca e análise dos artigos.
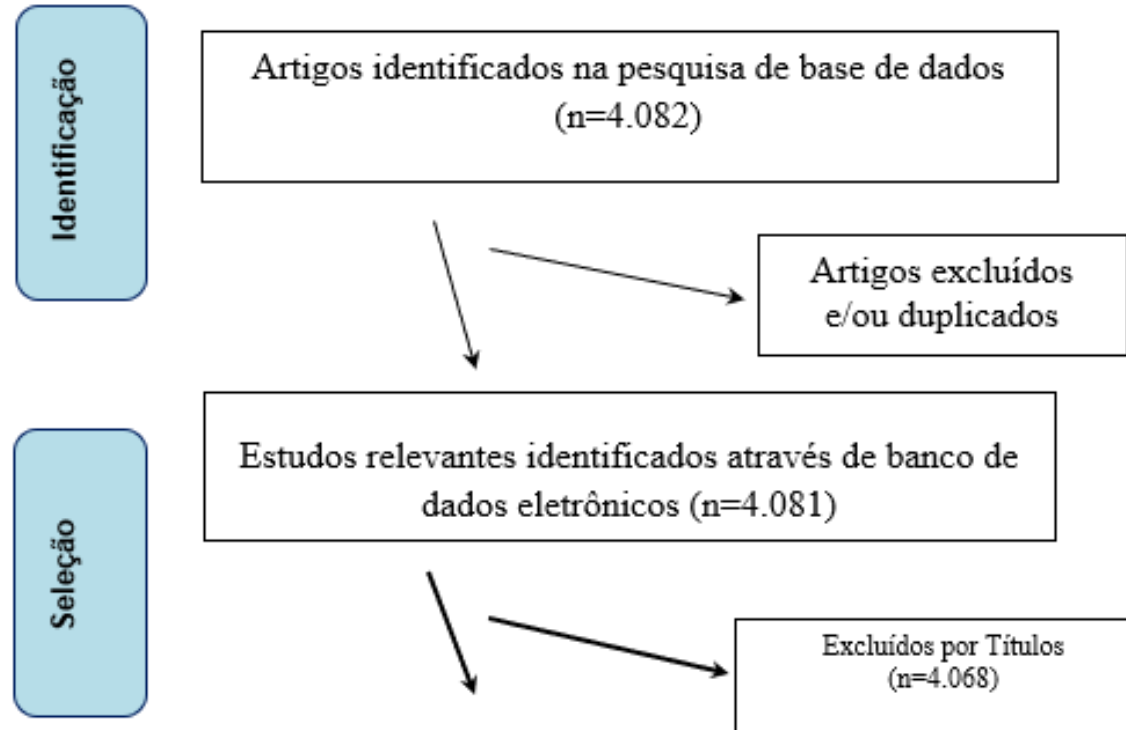

Estudos relevantes identificados através de banco de dados eletrônicos $(n=4.081)$
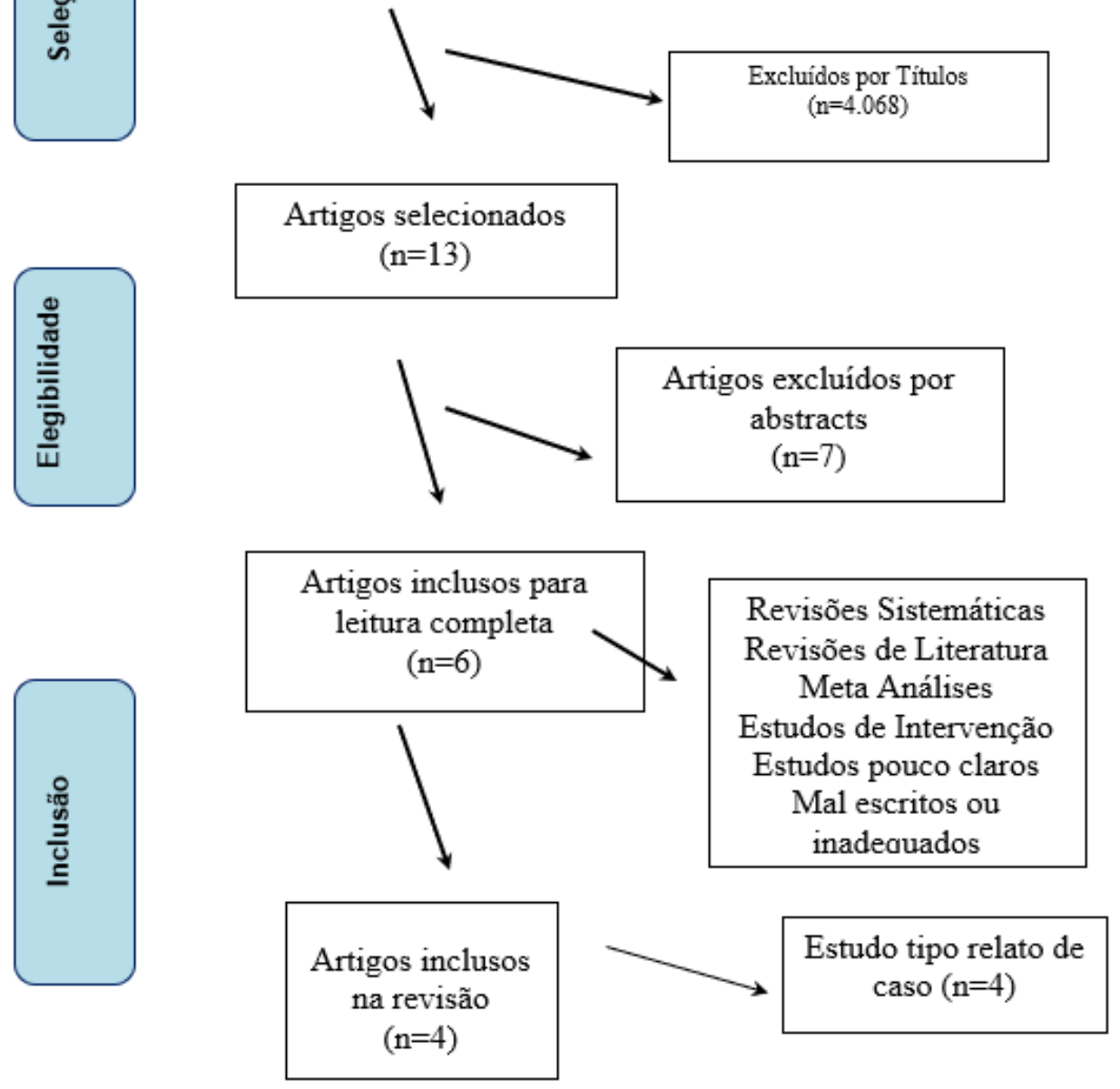

Fonte: Desenvolvido pelos autores

A partir dos descritores eleitos, os bancos de dados foram consultados e obtidos os resultados disponibilizados na Tabela 3. 
Tabela 3. Classificação das referências obtidas nas bases de dados Lilacs, Pubmed Science Direct, Scielo e Scopus.

\begin{tabular}{|c|c|c|c|c|c|}
\hline Descritores & $\begin{array}{c}\mathrm{N}^{0} \text { total de } \\
\text { artigos }\end{array}$ & $\begin{array}{c}\mathbf{N}^{\circ} \text { de } \\
\text { Referências } \\
\text { excluídas }\end{array}$ & Motivo da exclusão & $\begin{array}{l}N^{\circ} \text { de artigos } \\
\text { selecionados }\end{array}$ & Banco de dados \\
\hline $\begin{array}{l}\text { (Treacher collins Syndrome) or } \\
\text { (mandibulofacial dysostosis) or } \\
\text { (speech) or (maxillofacial } \\
\text { abnormalities) or (speech therapy) }\end{array}$ & 33 & 33 & Exclusão por título & 0 & Lilacs \\
\hline $\begin{array}{l}\text { (Treacher collins Syndrome) or } \\
\text { (mandibulofacial dysostosis) or } \\
\text { (speech) or (maxillofacial } \\
\text { abnormalities) or (speech therapy) } \\
2015-2020\end{array}$ & 1.153 & 1.152 & Exclusão por título & 1 & Pubmed \\
\hline $\begin{array}{l}\text { (Treacher collins Syndrome) or } \\
\text { (mandibulofacial dysostosis) or } \\
\text { (speech) or (maxillofacial } \\
\text { abnormalities) or (speech therapy) } \\
2015-2020\end{array}$ & 8 & 6 & Exclusão por título & 2 & Science Direct \\
\hline $\begin{array}{l}\text { (Treacher collins Syndrome) or } \\
\text { (mandibulofacial dysostosis) or } \\
\text { (speech) or (maxillofacial } \\
\text { abnormalities) or (speech therapy) } \\
\text { 2015-2020 }\end{array}$ & 2.888 & 2.887 & Exclusão por título. & 1 & Scielo \\
\hline $\begin{array}{l}\text { (Treacher collins Syndrome) or } \\
\text { (mandibulofacial dysostosis) or } \\
\text { (speech) or (maxillofacial } \\
\text { abnormalities) or (speech therapy) } \\
2015-2020\end{array}$ & 0 & 0 & 0 & 0 & Scopus \\
\hline Total & 4.082 & 4.078 & & 4 & $\begin{array}{c}\text { Pubmed, Scielo e } \\
\text { Science Direct }\end{array}$ \\
\hline
\end{tabular}

Fonte: Desenvolvido pelos autores.

As principais características dos estudos selecionados para esta pesquisa encontram-se expostas na Tabela 4. 
Tabela 4. Síntese dos artigos incluídos.

\begin{tabular}{|c|c|c|c|c|c|}
\hline $\begin{array}{c}\text { Autor/ Ano/ } \\
\text { Local de } \\
\text { publicação }\end{array}$ & Objetivo & $\mathbf{N}$ & Método & Resultados & Conclusão \\
\hline $\begin{array}{l}\text { Polanski, Plawiak } \\
\text { e Ribas. } \\
\text { São Paulo, } 2015 .\end{array}$ & $\begin{array}{c}\text { Descrever um } \\
\text { caso de } \\
\text { reabilitação } \\
\text { auditiva por meio } \\
\text { do uso de prótese } \\
\text { auditiva ancorada } \\
\text { no osso em um } \\
\text { paciente portador } \\
\text { da STC. }\end{array}$ & 1 & $\begin{array}{c}\text { Os resultados foram } \\
\text { avaliados por meio de } \\
\text { testes auditivos } \\
\text { comportamentais e dos } \\
\text { questionários Muss e } \\
\text { IT-Mais. }\end{array}$ & $\begin{array}{l}\text { A criança atingiu, um } \\
\text { mês após o uso da } \\
\text { PAAO, } 100 \% \text { de escore } \\
\text { no IT-Mais e conseguiu } \\
\text { direcionar a atenção à } \\
\text { fonte sonora, detectar e } \\
\text { reconhecer sons verbais } \\
\text { e reagir a ordens } \\
\text { complexas. }\end{array}$ & $\begin{array}{c}\text { Os testes audiológicos } \\
\text { posteriores, bem como as } \\
\text { avaliações de habilidades } \\
\text { de fala e de audição, } \\
\text { também demonstraram } \\
\text { melhores capacidades de } \\
\text { comunicação e audição. }\end{array}$ \\
\hline $\begin{array}{l}\text { Alfonso e } \\
\text { Centelles. } \\
\text { Cuba, } 2016 .\end{array}$ & $\begin{array}{c}\text { Apresentar um } \\
\text { estudo de caso de } \\
\text { uma família } \\
\text { cubana acometida } \\
\text { pela STC. }\end{array}$ & 3 & Exame físico e clínico. & $\begin{array}{c}\text { As malformações foram } \\
\text { confirmadas por meio } \\
\text { de tomografia } \\
\text { computadorizada, } \\
\text { também foi detectada } \\
\text { síndrome intra selar, e } \\
\text { ausência de } \\
\text { pneumatização das } \\
\text { células dos seios da } \\
\text { mastóide e esfenoidal. }\end{array}$ & $\begin{array}{l}\text { Embora a STC seja uma } \\
\text { doença congênita rara, é } \\
\text { importante conhecê-la para } \\
\text { um diagnóstico correto e } \\
\text { precoce, trazendo ao } \\
\text { paciente um tratamento } \\
\text { oportuno e multidisciplinar. }\end{array}$ \\
\hline $\begin{array}{c}\text { Hopper et al. } \\
\text { S.L, } 2018\end{array}$ & $\begin{array}{l}\text { Apresentar uma } \\
\text { técnica cirúrgica } \\
\text { para melhorar a } \\
\text { morfologia das } \\
\text { vias aéreas em } \\
\text { pacientes com } \\
\text { STC. }\end{array}$ & 5 & $\begin{array}{c}\text { Todos os pacientes } \\
\text { foram submetidos a } \\
\text { osteotomias sub } \\
\text { cranianas com } \\
\text { osteotomias } \\
\text { mandibulares } \\
\text { simultâneas, seguidas } \\
\text { de distração } \\
\text { maxilomandibular } \\
\text { coordenada com rotação } \\
\text { anti-horária. }\end{array}$ & $\begin{array}{l}\text { Houve uma recidiva } \\
\text { simétrica de } 30 \% \text { da } \\
\text { rotação com oclusão } \\
\text { mantida nos primeiros } \\
\text { nove meses de } \\
\text { acompanhamento que } \\
\text { então } \\
\text { estabilizado. }\end{array}$ & $\begin{array}{l}\text { A melhora resultante na } \\
\text { anatomia das vias aéreas } \\
\text { permitiu a decanulação em } \\
\text { quatro dos cinco pacientes } \\
\text { traqueostomizados. }\end{array}$ \\
\hline $\begin{array}{l}\text { Massi, Vieira, } \\
\text { Guarinello, Silva, } \\
\text { Tonocchi e } \\
\text { Wosiacki. } \\
\text { S.L, } 2019 .\end{array}$ & $\begin{array}{c}\text { Analisar o } \\
\text { processo } \\
\text { terapêutico } \\
\text { voltado à } \\
\text { oralidade de um } \\
\text { menino com a } \\
\text { síndrome de } \\
\text { Treacher Collins. }\end{array}$ & 1 & $\begin{array}{c}\text { Estudo de caso } \\
\text { longitudinal e } \\
\text { prospectivo realizado } \\
\text { em uma clínica escola. }\end{array}$ & $\begin{array}{l}\text { Os resultados mostram } \\
\text { que o paciente } \\
\text { progrediu em relação ao } \\
\text { desenvolvimento da } \\
\text { linguagem oral. }\end{array}$ & $\begin{array}{c}\text { A intervenção } \\
\text { fonoaudiológica em } \\
\text { questão tem contribuído } \\
\text { com o processo de } \\
\text { apropriação da linguagem } \\
\text { oral do paciente com STC. }\end{array}$ \\
\hline
\end{tabular}

Legenda: STC $=$ Síndrome de Treacher Collins. Muss = Meaningful Use of Speech Scale. IT-Mais = Infant-Toddler Meaningful Auditory Integration Scale. PAAO = Prótese Auditiva Ancorada no Osso.

Fonte: Polanski et al. (2015), Alfonso e Centelles (2016), Hopper et al. (2018), Massi et al. (2019). 


\section{Desenho dos estudos}

O primeiro estudo (Polanski, et al., 2015) teve como propósito analisar um caso de reabilitação auditiva por meio do uso de prótese auditiva ancorada no osso em um paciente de três anos acometido pela síndrome de Treacher Collins que apresentava problemas relacionados à síndrome especialmente no tangente à audição e vias aéreas superiores (VAS). Inicialmente utilizaram um aparelho auditivo em arco de vibração que deu ganho de $40 \mathrm{~dB}$, mas não houve boa aceitação por parte do paciente. Posteriormente foi utilizada uma prótese auditiva ancorada no osso Baha modelo BP100, com softband. 30 dias após a ativação do sistema Baha, foram aplicados testes auditivos comportamentais e os questionários Meaningful Use of Speech Scale e Infant-Toddler Meaningful Auditory Integration Scale.

O segundo estudo (Alfonso, et al., 2016) relata o caso de uma família portadora da síndrome de Treacher Collins, o paciente em questão era do sexo masculino, 22 anos e procurava por tratamento estético, o pai e a irmã também eram portadores da síndrome de Treacher Collins. Inicialmente foi realizado exame físico e posteriormente tomografia computadorizada simples de crânio e face que confirmaram as características sindrômicas.

O terceiro estudo incluído (Hopper, et al., 2018), teve como objetivo melhorar a morfologia das vias aéreas em pacientes traqueostomizados com síndrome de Treacher Collins. Participaram do estudo cinco indivíduos, sendo que três tiveram tubos de gastrostomia antes da cirurgia e os outros dois possuíam tubos de gastrostomia anteriores que foram substituídos no momento da cirurgia para auxiliar na nutrição pós cirurgia. Pacientes em fixação maxilomandibular (MMF) em torno de uma placa oclusal de acrílico customizada com pinos de tração extra oral de um arco facial embutido foram recrutados para o estudo. Os dispositivos de distração da mandíbula foram utilizados para conservar o contato dos côndilos com a base do crânio durante a rotação, mas foi tomado cuidado para garantir que a tensão máxima ocorresse nos fios do dispositivo e não nos pinos mandibulares para evitar compressão indevida na articulação temporomandibular (Hopper, et al., 2018).

O quarto estudo (Massi, et al., 2019), consistiu em analisar um estudo clínico longitudinal e prospectivo na área de fonoaudiologia, mais especificamente sobre a linguagem de um paciente acometido pela síndrome de Treacher Collins, atendido semanalmente em uma clínica escola de fonoaudiologia no Sul do Brasil. Os dados considerados para o estudo foram os atendimentos clínicos do paciente de 2012 a 2016, a coleta de dados (Massi, et al., 2019) registrou diversos pormenores do prontuário como a entrevista inicial com os pais, avaliações fonoaudiológicas, relatórios semestrais, devolutivas, gravações interativas entre o terapeuta e a criança. Vale lembrar para fins de registro que como se tratava de uma clínica escola, o paciente era atendido por terapeutas diferentes a cada semestre.

\section{Achados Fonoaudiológicos}

O resultado (Polanski, et al. 2015) do teste comportamental alcançou 100\% no escore do questionário Infant-Toddler Meaningful Auditory Integration Scale, o paciente alcançou a capacidade de direcionar a concentração à fonte sonora, perceber sons verbais e responder ordens complexas. Importante destacar que o paciente permaneceu em atendimento fonoaudiológico e médico de forma multidisciplinar.

No que se refere à audição, voz e motricidade orofacial do paciente no ano de 2012, na entrevista inicial, o paciente tinha ductos e pavilhões auditivos presentes embora tivesse a orelha média preservada, apresentava dificuldade respiratória e deglutitória, não fazia vedamento labial e tinha atenuação de fluxo aéreo nas vias aéreas superiores, as bochechas e olhos apresentavam assimetria, mandibular retrognática e ausência de movimentos de captação, preparação e mastigação do alimento (Massi et al., 2019).

Quanto à alimentação do paciente em 2012, o mesmo apresentava capacidade de deglutir apenas líquidos e no que tange a saúde e qualidade vocal do paciente, o indivíduo apresentava voz baixa. Em relação à linguagem do paciente, segundo relato da mãe, o mesmo se comunicava através de expressões faciais e mobilização de lábios e língua, apresentando dificuldades de 
comunicação com não familiares. Após 20 meses de terapia fonoaudiológica, o paciente conseguiu agregar sonoridade na fala, levando a mãe a relatar que agora ele se comunica com gestos, mas também fala enquanto gesticula. O processo terapêutico foi enfatizado em possibilidades interacionais do paciente com o seu meio (Massi, et al., 2019).

\section{Demais tratamentos}

Segundo os autores (Alfonso et al., 2016) o tratamento estético preferencialmente seria mais efetivo se realizado mais cedo, todavia os pacientes deste estudo apresentavam funções respiratórias, nutricionais e desenvolvimento cognitivo normal, o que tornou o prognóstico dessa família promissor, ainda que a intervenção estética não tivesse sido feita precocemente.

Hopper et al. (2018) demonstram que a distração mandibular osteogênica variou de 23 a 34 dias. Todos os participantes tiveram a consolidação das osteotomias mandibulares distraídas no momento da remoção dos dispositivos. Os pacientes 2, 3, 4 e 5 (adolescentes com reconstrução malar após-remoção) tiveram consolidação das osteotomias medianas da face, enquanto o paciente 1 (pré-adolescente sem malar enxertos) demonstrou recidiva rotacional da maxila e a necessidade de cirurgia reposicionamento e fixação da placa. Paciente 2, 3, 4 e 5 foram todos decanulados com sucesso com base no exame das vias aéreas e resultados da polissonografia. O paciente 1 não havia sido decanulado, aguardando a cirurgia planejada de redução da base da língua. A abertura interincisal foi comparável à pré-operação.

Quanto às complicações associadas à cirurgia (Hopper, et al., 2018), o paciente 1 teve abscesso na bochecha que foi resolvido com drenagem e recidiva no meio da face, necessitando de cirurgia de redução aberta e fixação interna. O paciente 3 também apresentou abscesso na bochecha e também foi resolvido com drenagem, além disso também teve abscesso orbital, resolvido com remoção do implante. Os demais pacientes não apresentaram complicações.

Na maioria dos estudos incluídos nesta revisão, foi destacada a importância da multidisciplinaridade no prognóstico da síndrome de Treacher Collins, que é um ponto reforçado por estudos como o de Lodovichi et al. (2018) que salienta que as pessoas nascidas com Síndrome de Treacher Collins precisam de tratamento longitudinal, com abordagem multidisciplinar. O estudo de Maidana, et al. (2020) também reafirma os problemas respiratórios por conta das vias aéreas difíceis comumente encontradas em pacientes com disostose mandibulofacial, sendo necessário até mesmo a análise individual dos pacientes em caso de necessidade de anestesia e intubação.

Quanto ao impacto da síndrome na audição, as pesquisas atuais mostram que as características sindrômicas comumente afetam a anatomia da orelha externa, como microtia (Lourençone, 2018). Existem alguns fatores como que tipo de deformidade e a gravidade, a deformidade da orelha ligada à síndrome de Treacher Collins é a atresia aural congênita que frequentemente causa perda auditiva condutiva, seja pela ausência de pavilhão auricular, obstruções do canal auditivo ou anormalidades na cadeia ossicular da orelha média (Lee et al., 2020). Consequentemente, a perda auditiva unilateral, poderá resultar em dificuldades de localização do som e compreensão da fala, sobretudo em ambientes barulhentos, causando danos nas interações sociais e aprendizado dos acometidos pela síndrome (Matos et al., 2020).

\section{Considerações Finais}

As características sindrômicas da disostose mandibulofacial afetam diretamente a audição, fala, voz, deglutição e respiração dos pacientes, sendo essas particularidades que tornam a atuação fonoaudiológica na equipe multidisciplinar de extrema importância para o prognóstico e manutenção da qualidade de vida dos pacientes acometidos pela síndrome de Treacher Collins. Destaca-se também, que pela escassez de estudos relacionados às características sindrômicas relacionadas à profissão de fonoaudiologia, mais pesquisas devem ser feitas aprofundando os aspectos da síndrome e o que a atuação fonoaudiológica poderia fazer como intervenção. A partir disso, nota-se a escassez na literatura sobre o tema, necessitando assim de trabalhos futuros que abordem mais detalhadamente sobre o assunto pertinente porém com poucos estudos sobre. 
Research, Society and Development, v. 10, n. 3, e49810313712, 2021

(CC BY 4.0) | ISSN 2525-3409 | DOI: http://dx.doi.org/10.33448/rsd-v10i3.13712

\section{Referências}

Alfonso, L. S., \& Centelles, I. A.,(2016). Síndrome de Treacher Collins en una familia cubana: presentación de caso. Revista Habanera de Ciências Médicas, $15(3), 408-417$.

Da Silva, R. C. T. (2017). Revisão Bibliográfica sobre a Síndrome de Treacher Collins: Descrições embriológicas e fenotípicas. Caderno de Graduação Ciências Biológicas e da Saúde, 4(1), 27.

Delgado-Maidana, W., Alonso, R., De Barros-Barreto \& Mazó AC (2020). Anestesia en paciente pediátrico con Síndrome de Treacher Collins. Mem. Inst. Investig. Ciencias de la Salud, 18(3), 67-72

Duque, C. L. (2019). Síndrome de Treacher Collins e implicações na cavidade oral. Dissertação (Mestrado) - Curso de Medicina Dentária, Faculdade de Ciências da Saúde, Universidade Fernando Pessoa, Porto, Cap. 3.

Hopper, R. A (2018). Counterclockwise Craniofacial Distraction Osteogenesis (C3DO) for tracheostomy-dependent children with Treacher Collins syndrome. Plastic And Reconstructive Surgery Advance Online Article, 142, 1-33.

Lee, M. Y. (2020). Current Treatments for Congenital Aural Atresia. Journal Of Audiology And Otology, 24(4), $161-166$.

Lodovichi, F. F (2018). Traz a deformidade de orelha impacto negativo na qualidade de vida em indivíduos com síndrome de Treacher Collins? Ciência \& Saúde Coletiva, 23(12), 4311-4318.

Lourençone, L. F. M. Prótese auditiva ativa cirurgicamente implantáveis de orelha média para reabilitação auditiva em pacientes com atresia aural congênita bilateral: técnica cirúrgica e resultado audiológico. Agência USP de Gestão da Informação Acadêmica (ÁGUIA), 188.

Massi, G., França, D. R., Santos, R. S., Ribas, A., Fonseca, V. D., Guarinello, A. C., \& Ziesemer, N. de B (2016). Speech language pathology findings in a Treacher Collins syndrome patient. The International Tinnitus Journal, 20(1), 31-35.

Massi, G., Vieira, S. K., Guarinello, A. C., Silva, A. P. B. V., Tonocchi, Rita; \& Wosiacki, F. T (2019). A linguagem na Síndrome de Treacher Collins: uma análise dialógica. Audiology - Communication Research, 24, 1-7.

Moher, D., Shamseer L., \& Clarke M. (2015). Preferred reporting items for systematic review and meta-analysis protocols (PRISMA-P) 2015 statement. Systematic Reviews, $4(1), 1$.

Pithon M. M., Sant'anna L., Baião F.C.S., Santos R.L., Coqueiro R.S., \& Maia L. C. (2015). Assessment of the effectiveness of mouthwashes in reducing cariogenic biofilm in orthodontic patients: a systematic review, 43,297-308.

Polanski, J. F., Plawiak, A. C., \& Ribas, A (2015). Reabilitação auditiva na Síndrome de Treacher Collins por meio de prótese auditiva ancorada no osso. Revista Paulista de Pediatria, 33(4), 483-487.

Saoud, A., Taïbi, B., Farouki, Aymane E., Chat, L., Haddad, S. E., \& Allali, N (2020). Treacher Collins syndrome: a case report. International Journal Of Case Reports And Images, 11, 1-5. 